\title{
Espiritualidad, religiosidad y enfermedad: una mirada desde mujeres con cáncer de mama
}

\author{
Spirituality, Religiosity and IIlness: a view from Women \\ with Breast Cancer \\ Espiritualidade, religiosidade e doença: uma olhada desde mulheres \\ com cancro de mama
}

\begin{abstract}
Yesica Milena Puentes Silva*, Sandra Carolina Urrego Barbosa*, Ricardo Sánchez Pedraza*
Grupo de Investigación Clínica, Instituto Nacional de Cancerología, Colombia
\end{abstract}

Doi: dx.doi.org/10.12804/apl33.03.2015.08

\section{Resumen}

La presente indagación buscó explorar el lugar de la espiritualidad y de la religiosidad en la manera como un grupo de mujeres con cáncer de mama asumen, significan y enfrentan su enfermedad. Para ello se efectuó el análisis cualitativo de una serie de entrevistas en profundidad realizadas a un grupo de cuatro mujeres participantes en una "intervención basada en espiritualidad" del Instituto Nacional de Cancerología. Tras un ejercicio de examen y confrontación teórica, se logró una aproximación a los modos de agenciamiento e interpretación de los eventos de la enfermedad, los cuales, a su vez, se ven cruzados por concepciones y prácticas relacionadas con "lo espiritual" y "lo religioso", así como por el saber médico. Las ideas de las mujeres sobre enfermedad y cuidado revelan una conjunción de estos elementos. Principales categorías emergentes: espiritualidad, religiosidad y enfermedad.

Palabras clave: espiritualidad; religión; enfermedad.

\section{fbstract}

The present investigation aims to explore the role of spirituality and religiosity in the way that women with breast cancer assume, signifiy and face their illness. For this, a qualitative analysis of a series of in-depth interviews with a group of four women participating in a "spirituality based intervention" of the Instituto Nacional de Cancerología, is made. After an exercise of review and theoretical confrontation, an approximation towards the modes of agency and interpretation of the illness was achieved, modes which are crossed concepts and practices related to the "spiritual" and "religious" area as well as the medical knowledge. The ideas of the

* Yesica Milena Puentes Silva y Sandra Carolina Urrego Barbosa, Grupo de Investigación Clínica, Instituto Nacional de Cancerología; Ricardo Sánchez Pedraza, Universidad Nacional de Colombia y Grupo de Investigación Clínica, Instituto Nacional de Cancerología

La correspondencia relacionada con este artículo debe ser enviada al Grupo de Investigación Clínica. Correos electrónicos: ympuentess@unal.edu.co, surrego@cancer.gov.co,rsanchez@cancer.gov.co

Cómo citar este artículo: Puentes, Y. M., Urrego, S. C. \& Sánchez, R. (2015). Espiritualidad, religiosidad y enfermedad: una mirada desde mujeres con cáncer de mama. Avances en Psicología Latinoamericana, 33(3), 481-495. doi: dx.doi.org/10.12804/apl33.03.2015.08 
women about illness and care reveal a combination of these elements. Major emerging categories: spirituality, religiosity and illness.

Keywords: spirituality; religion; illness.

\section{Resumo}

A presente pesquisa busca explorar o lugar da espiritualidade e da religiosidade na maneira como o grupo de mulheres com cancro de mama assumem, significam e enfrentam sua doença. Para isto, efetua-se a análise qualitativa de uma série de entrevistas em profundidade realizadas a um grupo de quatro mulheres participantes em uma "intervenção baseada em espiritualidade" do Instituto Nacional de Cancerologia. Depois de um exercício de exame e confrontação teórica, consegue-se uma aproximação aos modos de agenciamento e interpretação dos eventos da doença, os quais a sua vez são cruzados por concepções e práticas relacionadas com "o espiritual" e "o religioso" assim como pelo saber médico. As ideias das mulheres sobre a doença e cuidado revelam uma conjunção destes elementos. Principais categorias emergentes: espiritualidade, religiosidade e doença. Palavras-chave: espiritualidade, religião, doenças.

\section{Espiritualidad/religiosidad y enfermedad: algunos apuntes desde la perspectiva de las ciencias sociales}

La enfermedad, como experiencia vital, adquiere los visos de un evento existencial que despliega una serie de transformaciones en diversos aspectos: corporal, psíquico, emocional y social. Estas transformaciones, a su vez, requieren la constitución subjetiva de posibilidades de asimilación y de manejo, al igual que la movilización de distintos “capitales humanos" para la construcción de sentido y significado, recursos que abarcan lo material, lo intelectual y lo afectivo, y, por supuesto, derivan y se nutren de marcos socioculturales específicos. Es decir, de una panorámica que configura lo cosmológico, lo mitológico, lo estético, lo ético, lo político, lo económico. De este modo, vale la pena señalar para empezar que existe una línea de significación en la cual la enfermedad - en este caso, el cáncer de mama - adquiere matices verdaderamente especiales, y es la de los fenómenos religiosos.

Geertz plantea un "paradigma" a la hora de tratar el problema de la significación, en lo que siguiendo a Parsons y Shils, denomina dimensión cultural del análisis religioso (2005, p. 88). Consiste en afirmar la función sintética de los "símbolos sagrados" que recogen tanto el ethos de un pueblo —entendido como "el tono, el carácter y la calidad de su vida, su estilo moral y estético" (Geertz, 2005, p. 89) - como su cosmovisión —entendida como "el cuadro que ese pueblo se forja de cómo son las cosas en la realidad, sus ideas más abarcativas acerca del orden" (Geertz, 2005, p. 89)—.

La interacción entre estos dos órdenes produce efectos de doble vía que desembocan en una congruencia unitaria. De este modo, Geertz (2005) establece, por un lado, las prescripciones morales y estéticas como consecuencias "naturales" e inherentes de una estructuración particular de la "realidad"; por otro, otorga evidencia de la certeza de esta estructura de mundo acudiendo a sentimientos estéticos y morales, de gran poder emotivo y obviedad experiencial. De allí mismo surge la idea de religión del citado autor, un concepto integrativo de estas dos esferas.

La enfermedad, por su lado, no es ajena a este tipo de estructuración "religiosa" de la realidad, principalmente para quienes se adscriben a ella (aunque tal vez cumpla un papel referencial y eventualmente ejerza alguna influencia, ya sea en términos negativos, para quienes por el contrario se apartan de sus sendas). Y a partir de este tipo de estructuración es posible que la enfermedad se modele estética, ética y cosmológicamente, de acuerdo con ciertos parámetros, sin descontar la fuerza transformadora de la subjetividad en todos aquellos procesos.

Al decir de Bourdieu, la religión constituye "un sistema simbólico estructurado que funciona 
como principio de estructuración” (2006, p. 50), que "1. Construye" y " 2 . gracias al principio de consagración (o de legitimación) [...], hace sufrir al sistema de de las disposiciones respecto del mundo natural y del mundo social inculcadas por las condiciones de existencia, un cambio de naturaleza" (p. 50). Y, además, está "predispuesta a asumir una función ideológica, práctica y política de absolutización de lo relativo y de legitimación de lo arbitrario" (p. 50).

Así, la enfermedad puede aprehenderse tanto en términos ideológicos como en términos prácticos a partir de este principio estructurante (si no, al menos puede verse influenciada), y emprenderse la orientación de recursos materiales, afectivos e intelectuales según el criterio organizativo propio de una cosmovisión específica, así como ejerciendo una función expresiva por medio de la estética y la moral de un ethos particular.

Cierto es, como lo precisa Csordas (2004), que cuando nos referimos a la palabra religión, nos trasladamos a un denso y profundo bagaje histórico y cultural; sin embargo, la señalización de un punto de partida permite elucidar con mayor tranquilidad algunos rumbos.

Ya haciendo referencia a la "religiosidad" y a la manera como puede llegar a enlazarse con la enfermedad, el panorama resulta también bastante complejo; pero más aún si a ello se suma la idea de espiritualidad. Muchas veces, espiritualidad y religiosidad se usan como sinónimos o, por el contrario, se oponen. De esto se hablará en la siguiente sección, así como del concepto de espiritualidad, cuyo uso y enunciación se han abarcado principalmente desde disciplinas distintas a las ciencias sociales, como es el caso de las ciencias de la salud.

\section{Algunos apuntes desde la perspectiva de las ciencias de la salud}

En términos generales, desde la perspectiva de la psicología clínica - y más específicamente desde su expresión subdisciplinar de la psiconco- logía - se establece una distinción básica entre los conceptos de espiritualidad y religiosidad, que sigue, en general, la ruta de una distinción entre subjetividad e institucionalidad. Visser, Garssen y Vingerhoets (2010) revisaron los estudios llevados a cabo sobre la relación entre espiritualidad (distinguiéndola tanto teórica como metodológicamente de la religión y, por ende, de la religiosidad) y bienestar emocional (entendido como la ausencia de estrés, depresión, desesperanza, ideación suicida, y presencia de "calidad de vida", salud mental, adaptación, y funcionalidad social), y señalaron la existencia de diversos entendimientos de aquella, como la búsqueda de conexión y sentido o la experiencia subjetiva de lo "sagrado". A su vez, la espiritualidad es diferenciada de la "religión", al explicar que esta última ubica a la primera en el contexto de las creencias, de los valores y de las prácticas de una institución y, en consecuencia, identifica la religión con una forma específica de espiritualidad (Visser et al., 2010).

La metodología de esta revisión consistió en la búsqueda, la selección y el análisis de un conglomerado de textos (cuarenta) que reseñaban estudios alrededor de la relación entre espiritualidad o sentido de la vida y bienestar emocional. Como resultado, los autores señalan que en catorce de estos se estableció una discriminación entre una dimensión horizontal-existencial (entendida como un sentimiento de sentido, paz y conexión consigo mismo y con los demás) y una dimensión vertical-religiosa de la espiritualidad (creencia en la conexión con un "poder mayor"), que en su mayoría vincula positivamente la dimensión horizontal — no así la vertical — con el bienestar (Visser et al., 2010).

Otra concepción de espiritualidad, desde la perspectiva de Puchalski y Romer (2000), la define como un constructo que permite al individuo experimentar un sentido trascendente de la vida, que incluye los conceptos de fe y significado o sentido vital (Brady, Peterman, Fitchett, Mo \& Cella, 1999; Breitbart, 2002; Karasu, 1999). 
En contraste con lo anterior, otros estudios han hecho hincapié en la religiosidad. Es el caso del estudio de Zwingmann, Müller, Körber y Murken (2008), el cual refiere que la religiosidad consiste en una serie de actitudes, valores o prácticas vinculadas con un poder divino o "trascendente" (cosa también conocida a veces como espiritualidad), que se basa o no explícitamente en el sistema de creencias de una tradición religiosa (la más de las veces cristiana). Dicho estudio investigó la relación entre religiosidad y ajuste psicosocial, distinguiendo entre compromiso religioso disposicional (entendido como la importancia de la religiosidad en la vida propia, como la presencia de una disposición concebida como recurso) y el afrontamiento religioso en una situación específica (entendido como la manera en que se apoyan los "pacientes" en su fe religiosa en una situación crítica) (Zwingmann et al., 2008).

Mytko y Knight (1999), por su lado, hacen una revisión de la literatura concerniente a la relación entre espiritualidad/religiosidad, salud y "calidad de vida". Acá se asocia el término religioso con las prácticas y creencias propias de una institución, que son definitorias de modos específicos de ver la vida y que entrañan la búsqueda de lo "divino" (Mytko \& Knight, 1999). La espiritualidad, por otro lado, se refiere a esa misma búsqueda, pero a través de alguna "experiencia o ruta de vida" (Mytko \& Knight, 1999). Esta revisión se basa en el examen de las posibilidades psicométricas de varias escalas desarrolladas para "medir" la espiritualidad/religiosidad.

En la exposición de resultados de esta revisión se señaló la relación significativa entre espiritualidad/religiosidad, bienestar físico y psicosocial y "calidad de vida" (Mytko \& Knight, 1999). En resumen, se estableció que la espiritualidad y la religiosidad pueden otorgar mecanismos de afrontamiento de la enfermedad de diversa índole: cognitivos, afectivos, psicológicos y comportamentales. Los "pacientes" retrataron esto como un método importante que permitía sobrellevar la enfermedad (Mytko \& Knight, 1999).
Por otro lado, un equipo de investigación, conformado por un profesor en teología práctica y un grupo de especialistas en cáncer, investigan a partir de una metodología fenomenológica-hermenéutica - y de la realización de una serie de entrevistas en profundidad - el rol de la espiritualidad en la vida de mujeres con cáncer de mama para determinar si es esta benéfica o no en la tarea del afrontamiento y la mejora de la calidad de vida (Swinton, Bain, Ingram \& Heys, 2011). Para esto, y con el fin de orientar el ejercicio empírico, se inspiran en David Hay y Viktor Frankl, quienes elaboran (cada uno) un modelo de la espiritualidad que incorpora la religión, mas "no es definida por ella" (Swinton et al., 2011). Otros autores también apoyan esta teoría (Mytko \& Knight, 1999; Zwingmann et al., 2008).

En el caso de Hay, se trata de un entendimiento de la "espiritualidad" como conciencia relacional, la cual se experimenta cuando se cae en cuenta de una íntima interconectividad con los otros, con la divinidad (para quienes son creyentes) y con el mundo en el que se reside (Swinton et al., 2011). Frankl, por su lado, plantea una aproximación a la espiritualidad centrada en el significado (o en la búsqueda de significado) como propósito primordial del "ser humano", es decir, entiende la espiritualidad como voluntad de significar (Swinton et al., 2011). A partir de esto, distingue entre el significado último, el cual está relacionado con la "divinidad", y el significado próximo, que es eso que se ubica en un nivel temporal (Swinton et al., 2011).

Así es como, a partir del referente de estos dos modelos y el análisis de los hallazgos encontrados en las entrevistas en profundidad, los resultados apuntan a la ocurrencia de un "movimiento triple" en las mujeres entrevistadas: (a) un movimiento hacia adentro, que indica el trabajo introspectivo de la persona, así como una reevaluación de lo que ella es y de aquello en lo que debe centrarse en presencia de la nueva situación; (b) un movimiento hacia afuera, representado por un tipo de conciencia relacional, que capacita a la persona para advertir su ubicación dentro de una red de relaciones 
con posibilidades curativas, y (c) un movimiento hacia arriba, que propende a la búsqueda de un significado último, que para estas mujeres tiene que ver con su relación con "dios" y sus derivadas consecuencias (Swinton et al., 2011).

Otro grupo de autores con formación en distintas disciplinas - psiquiatría, sociología y antropología - ha examinado, por medio de entrevistas, los roles de la espiritualidad y la religión en el afrontamiento del cáncer de mama en mujeres chilenas y la manera en que la enfermedad los cambia (Choumanova, Wanat, Barrett \& Koopman, 2006). Las manifestaciones concretas de estos ámbitos se encontraron, por un lado, en la oración; esto es, en la dependencia en "dios" y su papel como guía a través de la enfermedad; por el otro, en el apoyo social que la "comunidad de fe" otorgaba a estas mujeres (Choumanova et al., 2006).

Por último, es importante señalar algunos datos que apoyan la necesidad de la presente indagación. En Colombia, el cáncer de mama es la tercera causa de muerte entre las mujeres después del cáncer de cuello uterino y el de estómago (Instituto Nacional de Cancerología \& International Agency for Research on Cancer, 2005; International Agency for Research on Cancer, 2008; Piñeros, Ferlay \& Murillo, 2006). En asociación con el diagnóstico de cáncer, se ha observado una importante comorbilidad psiquiátrica: se han estimado tasas de depresión clínicamente significativa en sobrevivientes de cáncer de mama (Fann et al., 2008; Hegel et al., 2006; Kim et al., 2008; Mehnert \& Koch, 2008); ansiedad, en un $26.5 \%$ de ellas (Morales, Robles, Jiménez \& Morales, 2007); síntomas de estrés postraumático, entre un 10 y un $11 \%$ (Hegel et al., 2006; Mehnert \& Koch, 2008), y malestar psicológico previo a la cirugía, en un 41\% (Hegel et al., 2006). Kissane et al. (1997) describieron un síndrome de desmoralización distinto a la depresión en pacientes terminales, caracterizado por desesperanza, pérdida de significado y deseo de muerte. Rousseau (2000) se refiere a tal crisis como sufrimiento espiritual.
Por ende, a la revisión presentada en esta introducción subyace la inquietud acerca de la forma en que las mujeres con cáncer usan su espiritualidad o religiosidad para asumir la enfermedad y enfrentarse a ella. Por esto se seleccionó un grupo de cuatro mujeres de un total que participó en un ensayo clínico diferenciado del presente estudio, denominado Intervención en espiritualidad en pacientes con cáncer de mama y realizado en el Instituto Nacional de Cancerología. Con su colaboración se llevó a cabo el presente estudio.

\section{Metodología}

\section{Población}

Cuatro mujeres de estratos dos y tres, en edades entre los 47 y los 61 años, dos de ellas con nivel de escolaridad técnico, una de ellas con educación secundaria y otra de ellas con escolaridad primaria, diagnosticadas con cáncer de mama en estadio avanzado que se encontraban participando en un ensayo clínico del Instituto Nacional de Cancerología, titulado Evaluación de la eficacia y la efectividad de una intervención basada en espiritualidad en pacientes con cáncer de mama: ensayo clínico aleatorizado - diferenciado del presente estudio - y a quienes se les solicitó su aprobación para participar en una serie de entrevistas en profundidad sobre la temática ya referida.

\section{Procedimiento}

Se estructuró un abordaje metodológico basado en investigación cualitativa, usando en concreto el método de entrevistas en profundidad. Estas se realizaron tras el consentimiento informado de las participantes. Cada entrevista tuvo una duración aproximada de hora y media, tiempo durante el que se indagó acerca de asuntos como la religiosidad, la espiritualidad y su relación con la enfermedad. Así mismo, el material fue grabado en audio tomando anotaciones escritas relevantes para la interpreta- 
ción de los contenidos (por ejemplo, respuestas matizadas emocionalmente). Las siguientes son algunas de las preguntas realizadas durante las entrevistas:

- ¿Cómo ha sido su relación consigo misma y con la vida antes de la enfermedad y después de esta? ¿Ha cambiado en algo?

- ¿Se considera una mujer espiritual? ¿Cómo vive la espiritualidad?

- En el ámbito familiar, ¿cómo es la vida espiritual?

- ¿Separa el ámbito espiritual del religioso o considera que son lo mismo?

- ¿Practica usted alguna religión en especial? ¿Asiste a alguna iglesia u organización religiosa? ¿En qué consiste esa práctica?

- ¿Cree usted que hay alguna distinción entre una práctica espiritual individual y una grupal? Si es así, ¿alguno de estos ámbitos tiene mayor importancia para usted?

- ¿Cómo ha sido su vida espiritual antes de la enfermedad y después de ella? ¿Ha cambiado en algo?

\section{Metodología de análisis}

Se utilizó un enfoque temático inductivo cuyo fin era buscar que el material obtenido se convirtiera en generador de temáticas y conceptos. El análisis se realizó mediante una identificación de las temáticas y categorías que fueron validadas por un experto en el tema (investigador del Departamento de Antropología de la Universidad Nacional de Colombia); posteriormente, se llegó a un consenso para definir los temas relevantes que respondían a los nexos entre espiritualidad, religiosidad y enfermedad para la obtención del presente artículo. Se intentó reflejar la veracidad de los datos mediante el uso de procedimientos iterativos (lectura inicial del material, identificación y generación de temas) y la transcripción literal de fragmentos del material aportado por las entrevistadas.

\section{Resultados}

Para cada uno de los temas y categorías ubicados se presentan comentarios, síntesis y fragmentos de transcripción de las entrevistas que pretenden sustentar la interpretación alcanzada. Algunos de estos fragmentos se editaron (por ejemplo, se suprimieron partes relacionadas con otros temas) para facilitar su lectura y enfocarla en los puntos centrales. Las identidades de las entrevistadas se resguardaron bajo seudónimo. Finalmente, los resultados se presentan agrupados en tres apartados, de acuerdo con la tematización que se practicó a partir de las transcripciones: (a) espiritualidad/religiosidad y el encuentro con la enfermedad; (b) ¿espiritualidad versus religiosidad o espiritualidad-religiosidad?, y (c) entendiendo la enfermedad: visiones de las mujeres partir de la espiritualidad-religiosidad.

\section{Espiritualidad/religiosidad y el encuentro con la enfermedad}

Una modalidad recurrente de construcción de sentido en la enfermedad está representada por los ámbitos de lo espiritual y de lo religioso en la vida de las mujeres participantes de la presente indagación. La enfermedad - como ocurrió con la mayoría - se entendió en términos de ruptura, al ser concebida como un punto de quiebre inminente. Sin embargo, una de ellas no expresó - o, al menos, no quiso expresar - un entendimiento "divisorio" de la irrupción de la enfermedad. Adelina manifestaba sobre posibles cambios en su relación consigo misma y con la vida a raíz de la enfermedad: "No, más bien no... No porque yo soy de las personas que yo creo mucho en Dios y sé que uno vino a este mundo para desencarnar cualquier día, que lo llame mi Dios, ¿cierto?”. De tal modo adoptaba una disposición a la conformidad, ante cualquier desenlace que por "voluntad divina" se pudiera presentar.

El tema de la espiritualidad/religiosidad pareciera menguar el sobresalto existencial generado 
por el encuentro con la enfermedad. Al canalizar dicho sobresalto en términos de una continuidad marcada en la subjetividad, se pasaría a subrayar otros de sus puntos afirmativos, incluso en detrimento del choque generado por tal encuentro. Además, al entender esa irrupción en virtud de una inevitabilidad primaria auspiciada por el papel de la "divinidad", se podría desechar algo de su carga emocional, de modo que aunque su expresión se diera en términos más bien negativos el estilo de "afrontamiento diferido" - concebido por Pargament como la acción conjunta con la divinidad para el afrontamiento de la enfermedad (Nairn \& Merluzzi, 2003) — podría ser ilustrativo de una estrategia en este sentido. La enfermedad, aquí referida como una realidad subjetivamente encarnada, en la posible pérdida de ese carácter encarnado como desenlace su procedencia, pasa a ser entendida como un designio de la propia "divinidad". De este modo pareciera que la enfermedad llegara a detentar un particular carácter "religioso-espiritual".

En síntesis, algunas mujeres llegaron a considerar la enfermedad en términos de ruptura; otras no. Sin embargo, el tema de la espiritualidad/religiosidad cruzó indistintamente el encuentro de todas las mujeres con aquella (ya se consideraran "espirituales" o no, antes de su aparición). Para todas, la espiritualidad/religiosidad resultó un ámbito clave tanto en el encuentro con la enfermedad como en la subsecuente vivencia de esta. Blanca lo expresaba así:

En el momento, como te lo digo, fue un impacto violento... Yo ya casi tengo un año y... y estoy siguiendo adelante yo... no me dejé vencer. Yo creo mucho en Dios, y... le pedí que me ayudara, que habían otras personas que se curaban, ¿por qué yo no me iba a curar?

Ella, desde su ingreso a la Iglesia Adventista del Séptimo Día - unos años atrás—, se había vinculado a un ejercicio de "obra misionera", pero aclaraba que no era tan "arraigado" como luego de la enfermedad. A partir de esta escudriñaba fervientemente "las escrituras" y se reunía con compañeros de su iglesia. "La iglesia es como un hospital de enfermos espirituales", señalaba, y agregaba que como novedad sentía tener más tiempo para dedicarse a "dios".

El símil de Blanca no deja de resultar sugestivo en términos de lo que refiere a una idea social de lo saludable vinculada a un determinado nicho institucional, el cual se hace portador de unas asunciones y prescripciones sobre del cuidado de sí en un contexto de enfermedad. Este nicho institucional, ya sea la iglesia o el hospital (entendidos como categorías genéricas), no solo prescribe modos de ser-actuar, sino que además constituye marcos referenciales para interpretar. Utilizando la idea de Geertz (2005) acerca de la capacidad de los símbolos sagrados para sintetizar el ethos y la cosmovisión, y extendiendo este planteamiento a los símbolos en general, el recurso literario de Blanca, tomado como una representación cultural, denota una efectividad sintética que plasma dos dimensiones del cuidado: la corporal y la espiritual.

Estas "dimensiones" interactúan y se constituyen mutuamente sin llegar a excluirse o reducirse a su tratamiento en uno de los nichos. De este modo, el cuidado en la iglesia y en el hospital cruza el cuerpo en la enfermedad de una manera particular: no solo al decir cosas sobre este, sino al motivarlo en prácticas. En el siguiente aparte del relato de Adelina (que surge cuando respondía a la pregunta sobre la posibilidad de considerarse una mujer espiritual) se observa algo de lo anterior:

Yo me considero muy espiritual, y eso es lo que a mí me ha ayudado tal vez muchísimo porque yo allá [en el hospital] hablando con la gente que va a la misma situación... Yo los veo tan deprimidos y tan decaídos, y como que esa enfermedad se los va llevando... Entonces yo creo que sí, la fe es muy importante en el ser humano, saber que existe un 
Dios y que él es el que lo saca a uno de esto [...] Es que, prácticamente digamos que la sanidad de esa enfermedad está en eso, no es ni la droga...

Adelina, quien se definía como católica pero eventualmente asistía a una iglesia "cristiana", ${ }_{1}$ señalaba además que antes de la enfermedad había "vacíos" en su "vida espiritual". Con la enfermedad, en cambio, todos los días se disponía a "orar" como parte de una preparación para "desencarnar", y velar por lo que concebía como un "sustrato vital" que acusaba un lazo con la divinidad. Para ella, esta preparación vino a constituir un ejercicio de cuidado de "esa parte que lleva desde que nace hasta que muere y no cuida".

Cecilia, por su lado, comentaba lo siguiente en relación con el papel de la espiritualidad/religiosidad en su vida al momento de la enfermedad: "No era, yo no era espiritual, qué hace que esto... Me acogí al señor, y por el señor es que yo voy bien [...] uno dice soy católico, apostólico y romano, pero solo palabras, mamita, porque de hecho...".

A partir de la enfermedad, Cecilia empezó a involucrarse junto con su familia (al igual que Adelina) en actividades como la "oración". Las dinámicas del hogar cambiaron en este sentido: se hacían reuniones para dicho propósito. La espiritualidad/religiosidad en la vida de Cecilia pasó a detentar un papel preponderante no solo en términos de su propio "acercamiento" con lo "divino", sino en términos del establecimiento de unos núcleos de confluencia familiar, ligados a dichos ámbitos como forma de afrontar la enfermedad. Del movimiento triple de la propuesta de Swinton et al. (2011) se puede apreciar en este punto uno de sus movimientos: el movimiento hacia afuera, que precisaba en la mujer la conciencia de una red de relaciones sociales con "potencial curativo" expresada en la confluencia familiar alrededor de la oración.
Sin embargo, en el caso de Cecilia, no solo se dio este movimiento, también se dio el movimiento hacia adentro, cuando, a partir de la enfermedad, ella llegó a establecer un "cambio de ruta", cruzado por la espiritualidad/religiosidad en su trayectoria vital. Finalmente, el movimiento hacia arriba -entendido como la búsqueda de un significado último y la relación de las mujeres con la "divinidad"también se vio alterado:

En cambio, ahorita con eso [la enfermedad]... no, uno $[\ldots]$ no falta a su misa y primero es cuestión de que jumm, por allá cada uno en su rancho, y eso dizque católico [...]. Ahora sí muy cerca con Dios, muy presente, muy cerquita en mi vida.

Con Blanca ocurrió lo mismo: también se presentaron los tres movimientos. El movimiento hacia adentro, en el que se reevaluaban viejas prácticas y se propendía a transformaciones "internas" y "externas"; el movimiento hacia afuera, especialmente en relación con su "comunidad de fe" - o en términos de Durkheim, con su "comunidad moral" (Bourdieu, 2006) - , y, finalmente, el movimiento hacia arriba, en el que se dio la intensificación de sus relaciones con la divinidad, lo que según ella se expresaba en una lectura intensa de los textos bíblicos.

Finalmente, el movimiento hacia arriba también se presentó en Marlén de una manera particular: incidió en su asistencia a una actividad nueva para ella, la "oración de sanación":

[...] a mí me han puesto manos, y uno siente, siente como la presencia de Dios. [...] Siento... cuando a mí me... me están orando, me colocan las manos el predicador. Y ora, ora tan fuerte que uno, a mí por ejemplo me primero me pongo que sudo, sudo... y después yo, yo tambaleo, ¿cierto?, [...] como que le llega algo al corazón, como se

1 La expresión iglesias cristianas comenzó a ser utilizada en Colombia en razón del monopolio religioso católico, para designar otras prácticas religiosas como el protestantismo y el pentecostalismo. Willems señala que la emergencia de estas nuevas prácticas se generó como una forma de cuestionar el orden católico establecido (citado por Beltrán, 2010). 
le... como que le entra algo... uno siente, que sí le está haciendo como efecto.

Vale agregar que acá el cuerpo es tomado por las disposiciones estructurantes del ejercicio de fe dentro de una comunidad moral, que fundada en un sistema simbólico responde a cierta materialidad; no solo en la medida en que este sistema se apropia de ella conceptualmente, sino en el sentido de que tal ejercicio ocurre para sus participantes en un marco de efectividad.

\section{¿Espiritualidad versus religiosidad o espiritualidad-religiosidad?}

Para Blanca, Cecilia y Marlén, la distinción entre espiritualidad y religiosidad estaba más bien desdibujada. Cecilia y Marlén, por su lado, entendían los dos ámbitos como la misma cosa. Blanca lo expresaba del siguiente modo:

Es que uno tiene que ser espiritual, ¿sí?, el ámbito religioso lo lleva a la espiritualidad. A tener más contacto con Dios, con Jesús... que son los únicos... y el espíritu santo. [...] Entonces... eso lo hace a uno más... lleno de espiritualidad... de comprensión.

Por su lado, Adelina comentaba:

No es lo mismo. Una cosa es lo espiritual, lo que uno lleve dentro su corazón [...] las acciones con las que haga uno [... ] a lo religioso, lo religioso digamos que... es una estatua, un estampado, una vitela... eh ¿sí?, que llegan allá y que rezan el padrenuestro [...] y salieron en las mismas, ¿cierto? (Risas).

A veces, las prácticas en estos ámbitos también podían presentar un carácter individual o colectivo en su ejecución. Blanca comentaba:

Pues es que uno tiene que... no solamente estar solo, sino también estar en comunidad [...] Y pedir- le mucho a Dios para que esos hijos se conmuevan (risa) [...] A veces les hablo de mi enfermedad. Y de cómo yo la he soportado, de [?], porque uno se entrega más espiritualmente a Jesús, porque Jesús hizo tantos milagros aquí en este mundo [...] Esos milagros los puede hacer con uno mismo ¿no?

En Blanca, la expresión de la espiritualidad-religiosidad se da en una forma particular. Antes de entrar a su iglesia, ya acompañaba una suerte de "obra misionera"; no obstante, con la enfermedad no solo se intensificó su relación "íntima" con la "divinidad", sino que además se arraigó su deseo por llevar el mensaje religioso de su comunidad moral a terceros. La idea de Visser et al. (2010) sobre la dimensión horizontal existencial, referida a un sentido de paz con los otros, y la de la dimensión vertical religiosa, referida a un vínculo con un "poder mayor", parece condensarse aquí en un especial sentido de conexión con lo divino, pero a través de una relación con los otros. Dicha relación es mediada por su contribución como laica a la reproducción y difusión de lo que llamaría Bourdieu (2006) los bienes de salvación, los cuales - siendo constitutivos del "interés religioso" de grupos particulares - en este contexto estarían referidos al carácter religioso-espiritual especial detentado por la enfermedad. Ello, a su vez, permitiría la efectiva transmisión de dichos bienes hallando en el cáncer una fuente de inspiración que supliera su condición de laica.

Finalmente, y retomando propiamente el punto de partida acerca de la manera en que las mujeres entienden y aplican categorías como espiritualidad y religiosidad, cabe señalar la presencia de una conjunción de elementos que abarcan ideas y prácticas rituales, morales y de pensamiento mítico-religioso, que resulta en un complejo interpretativo amplio. Tal es el caso de la relación estrecha entre religiosidad y espiritualidad que manifestaba Blanca, en la cual, a través de la primera, se entraba en contacto con figuras mítico-religiosas como el Espíritu Santo y Jesús, enlazándolos, al mismo 
tiempo, con la espiritualidad; o, como en el caso de Adelina, quien distinguía acentuadamente entre "religiosidad" y "espiritualidad", asociando la espiritualidad con las prácticas morales ("lo que uno lleve dentro su corazón [...] las acciones con las que haga uno"), y a la religiosidad con prácticas eclesiales y rituales.

\section{Entendiendo la enfermedad: visiones de las mujeres partir de la espiritualidad- religiosidad}

La interpretación de la enfermedad, así como el encuentro con esta y su ulterior vivencia, ha sido cruzada de manera importante por la espiritualidad-religiosidad en estas mujeres. Así lo expresaba Adelina:

El ser humano lo hizo Dios, no lo hizo el diablo [...] entonces Dios cuida de esa parte, y de esa esencia del ser humano, en su niñez. Ya después cuando ya viene el libre albedrío entonces ya cada uno hace lo que quiere. Entonces yo digo que... uno no se debe separar, desde pequeño, de esa partecita, de esa esencia, y es lo que uno siempre se separó... ¿Cierto que sí? Y es por eso que yo pienso que también vienen las enfermedades, vienen los problemas, vienen esas cosas...

Ella se explicaba el cáncer, en parte, sobre la base de esa gran ruptura, aunque también añadía otros factores:

Digamos que gente que... una maldición, un no sé qué, no, eso no es [...] Que es generativo, de pronto algunas personas, pero en otras no es de generación, digamos que, que el abuelo tuvo, el Tatarabuelo, o esas cosas... no, porque hay gente que no tuvo eso y resultó uno de los familiares en esa situaciones, entonces no viene de allá [...] El medio ambiente, es una de las problemáticas de esas enfermedades [...] La alimentación... todas esas cosas son las que han influido para que las células
[...] no sean fuertes, sino cualquier cosa las atrofia, las mató, las dañó [...] Pero que la parte espiritual es muy fundamental para que el ser humano pueda resistir y pueda aguantar, digámoslo así, aguantar... todos esos golpes que la vida le da.

La interpretación del cáncer en Adelina, por un lado, toma cierta inspiración de la cosmovisión propia de su comunidad moral — la Iglesia católica en su manifestación concreta en estas geografías-, la cual viene a trazar "concepciones de un orden general de la existencia", que permiten establecer relaciones entre la dimensión de lo espiritual y la de lo corporal. Esta cosmovisión, además, corresponde a un ethos determinado, entendido en términos geertzianos (2005). De este modo, Adelina puede concebir una "esencia" espiritual que se separa de su "centro divino", al momento del "libre albedrío", cosa que trastoca el apego a cierta normatividad valorativa haciendo su cuerpo propenso a la enfermedad. El cuerpo es cruzado aquí por el influjo de la religión, en cuanto sistema de símbolos o incluso en su papel de ciencia práctica (Geertz, 2005). Sin embargo, como se señaló, el discurso de Adelina resulta más complejo cuando se entra a considerar su renuencia hacia la "práctica religiosa" — particularmente hacia la práctica ritual—, de modo que en su interpretación de la enfermedad solo se presenta un influjo parcial de la doctrina religiosa institucional, pues esta, como se ha visto hasta ahora y se seguirá viendo, es nutrida también por el influjo del saber médico.

Blanca coincide en algunos puntos con la concepción de Adelina, sobre todo en lo que refiere a prácticas sobre el cuerpo y conductas alrededor de la salud, como en el caso de la alimentación. También en lo que refiere a la liberación de toda responsabilidad directa por parte de la divinidad:

Hay muchas personas que culpan a Dios por la enfermedad, aquí me lo han dicho [hospital]. Entonces uno dice [...] que uno no puede culpar 
a Dios, que ver mis errores de alimentación, de muchas cosas que uno hace en contra de su salud, que... que bueno las hizo y ya se arrepintió pero que quedan las secuelas, y que posteriormente viene la enfermedad, pero ¿cómo voy a culpar a Dios si Dios es amor?, y quiere que todo mundo se salve y que no tengamos ningún problema $[\ldots]$ qué tristeza sería que Dios... le, le mandara a uno esa enfermedad, no... Es uno mismo que se la ha buscado [...] Nosotros... como, como personas humanas llenas de defectos pues hacemos cosas incorrectas que más tarde vamos a pagar las consecuencias. Entonces para mí es eso. Yo le decía eso a la psiquiatra, que podía... podía ser porque no tuve hijos... la enfermedad, pero por mis errores. Mi manera de comer, de malcomer [...] entonces eso puede ocasionar la enfermedad.

Sin embargo, Blanca también podía entender la enfermedad para algunos efectos en términos de "direccionamiento divino". Cuando alguien no está muy bien encaminado en las "rutas escogidas": "Dios permite, que uno tenga esa enfermedad para ver si cambia... la manera de ser. Porque Él nos quiere y Él como un padre disciplina, corrige", señalaba. Pero al mismo tiempo, de manera significativa, asociaba los errores que la "divinidad" corregiría con algunas nociones "médicas" y "nutricionales" provenientes de las ciencias de la salud, acerca de los hábitos alimenticios.

No obstante, tanto para Adelina como para Blanca, el papel divino en la aparición de la enfermedad, aunque presente, no era leído en términos de una intervención directa. En el caso de Blanca, según ella, dios "permite" la aparición de la enfermedad como un modo de direccionamiento, y esta llega como efecto de errores en los "modos de ser", que pueden manifestarse en la forma del abordaje de caminos "moralmente incorrectos", así como en la ejecución de prácticas "poco saludables". Para el caso de Adelina, el sustrato espiritual que compone al "ser humano" y lo mantiene en asociación con lo divino, sufre una ruptura irreparable que deja al cuerpo en estado de vulnerabilidad. Sin embargo, para ambas, la irrupción de la enfermedad nunca obedece a un designio divino directo, a un acto voluntario, consciente e intencionado de la divinidad sobre sus cuerpos; la divinidad tan solo llega a actuar como un catalizador que permite su ocurrencia, y que en la transición de dicha aparición hacia el proceso de asimilación y vivencia pasa de detentar un rol pasivo y distanciado a convertirse en el regente primario de toda dirección.

Este punto de vista sobre el papel de la divinidad en la aparición de la enfermedad es igualmente compartido por Cecilia y por Marlén. Así lo expresaba Marlén:

Dios, no... creo. Para mí, no sé si estaré equivocada o no, yo creo que pues Dios, no le manda a uno las cosas así, ¿cierto? Esos son... cosas del destino, la vida... porque por decir, todo el mundo tenemos el cáncer, sino unos no se nos ha desarrollado, con cualquier cosita...

Y en palabras de Cecilia: "Dios no castiga, Dios no es un Dios de castigos. Dios es un Dios real y vivo, la vida la tenemos todos, nos la dio a todos. Él es un Dios de todos". Ella, además, compartía la visión de Blanca acerca del entendimiento de la enfermedad como punto de partida para un cambio de ruta vital. Así lo expresaba:

Para mí fue como un alto en el camino, como decirme por aquí vas mal Cecilia, toma este camino [...] eso yo era rumba, farra, paseo... vida chévere $[. .$.$] Para mí fue como un alto a decirme:$ “Quietica aquí, Margarita, que tu camino es este. Por aquí vas mal".

Y este cambio de ruta en ambos casos está cruzado por prescripciones propias tanto de la dimensión "espiritual" como de la "corporal" (prescripciones religiosas y médicas), además de estar, para estas mujeres, respaldado y acompañado por la divinidad. 
La idea de Mytko y Knight (1999) acerca de la separación entre religiosidad y espiritualidad, entendiendo la primera como la búsqueda de lo divino a través de las prescripciones de una determinada institucionalidad, y la segunda como esa misma búsqueda pero por medio de una "experiencia o ruta de vida" particular no deviene operativa en este aspecto. La "ruta de vida" que advierte Cecilia, por ejemplo, está atravesada por los constreñimientos de los sistemas simbólicos de la religión $\mathrm{y}$, así mismo, del sistema médico en el contexto del cáncer, cuando asocia, por un lado, dicha ruta con la orientación divina y, por el otro, la caracteriza en virtud de la dejación de prácticas que desde su perspectiva - pero tomando inspiración de nociones médicas del cuidado- interferían con su salud. Estos sistemas, al menos de manera parcial $-\mathrm{y}$ en una relación multilateral y dinámica junto con otros aspectos - condujeron a definir sentidos y posibles alternativas respecto de la manera como Cecilia llegó a entender tal "transformación". Las ideas alrededor de esta nueva "ruta de vida" fueron guiadas e inspiradas por las disposiciones simbólicas de estos sistemas, que desembocaron también en modos de acceso a "lo divino" (así como en "prácticas alrededor de la salud"), que obraron con efectividad material para sus agentes.

Volviendo a Adelina, para quien sí existía una diferenciación clara entre espiritualidad y religiosidad, ella encontraba lo siguiente acerca de la práctica formal o ritual del catolicismo:

No es necesario, ni salva a nadie. Uno debe ser una persona que hace buenas obras [...] obras de caridad [...] cosas así. No haga mal al prójimo, no hable de esa persona. Esas cosas así, que son las que hacen daño [...] eso es lo que uno debe evitar, cuando ya tiene esa parte espiritual que realmente le dice a uno qué es lo que debe y qué no debe hacer $[\ldots]$ uno no necesita ir donde los curas, donde los pastores, esa la vida y eso es lo real. [...] Y sin embargo uno hace todas esas esas cosas, bueno y sin embargo se enfermó [...] Al diablo no gusta que alguien adore a Dios. El diablo de una u otra forma hace zancadilla para que reniegue de ese Dios que tanta fe le tiene [...] Él no lo permitió [Dios] pero el diablo es más sagaz que cualquier otro, él no lo permitió; pero sin embargo le tocó a uno enfermar [...] Pero si uno tiene fe, hay mucha gente que se ha sanado por pura fe.

Adelina esboza aquí una segmentación entre una práctica moral y una práctica ritual (o formal) del catolicismo cuando se refiere, por un lado, a las "obras de caridad" y, por el otro, al vínculo con lo eclesiástico, y establece un lazo entre dicha práctica moral y lo espiritual; mientras que, a su vez, lo niega con respecto de la práctica ritual. Por otro lado, Adelina, a diferencia de Blanca, expresó de manera textual la no permisión de la divinidad en la ocurrencia de la enfermedad, pero finalmente ambas apuntaban a lo mismo: a la liberación de la divinidad de toda responsabilidad en el acontecimiento de irrupción de la enfermedad, aunque pusieran el énfasis en fuentes de causalidad parcialmente distintas (las trampas de lo que Adelina llamó el "diablo", que remitían a negaciones de la divinidad aun en el cumplimiento de todo precepto moral; y en el caso de Blanca, la desviación comportamental que incluyera los hábitos en salud y demás, lo que, sin embargo, también compartía Adelina).

\section{Discusión}

Las ideas de las mujeres entrevistadas con respecto del papel de la espiritualidad/religiosidad en su enfermedad reúnen, al mismo tiempo, cruces, simetrías y divergencias. En todos los casos, sin embargo, la espiritualidad/religiosidad ha resultado fundamental tanto en el momento de aparición de la enfermedad como en su subsecuente evolución. Es importante tener en cuenta que la espiritualidad/ religiosidad no solo brinda un marco interpretativo para la enfermedad, sino que estimula toda una 
serie de prácticas en el contexto del cáncer, por ejemplo, el ejercicio de oración que realiza Adelina en busca de "preparación espiritual".

En un caso como este, puede ser útil la indagación foucaultiana alrededor de las relaciones entre el sujeto y los juegos de verdad, las cuales inicialmente se develaban en virtud de las prácticas coercitivas (como el sistema penitenciario y la institución psiquiátrica) y las formas de juego teóricas o científicas (como el estudio de las riquezas, el lenguaje y el ser vivo) y que - a partir de los cursos que el autor dictó en el colegio de Francia - pasaron a ser vistas, a través de lo que denominó las prácticas de sí (Foucault, 1999). Para Foucault, estas prácticas tomaban la forma de una "práctica ascética" no tanto expresada en términos de renuncia, sino más bien entendida como "un ejercicio de uno sobre sí mismo, mediante el cual intenta elaborarse, transformarse y acceder a cierto modo de ser" (1999, p. 394).

Así es como el marco cosmológico y el ethos (Geertz, 2005), propio del catolicismo, estimula el ejercicio de unas prácticas de sí, las cuales - según lo sugieren Becker, Betancourt y Müller, en su diálogo con Foucault - manifiestan las relaciones entre subjetividad y verdad; pero en un nuevo terreno: el de la autotransformación en el ejercicio cotidiano (Foucault, 1999). Lo anterior resitúa y pone el relieve de las relaciones subjetividad-verdad en el campo de una serie de prácticas que son agenciadas y tramitadas por el sujeto mismo, y no por el influjo de prácticas coercitivas externas. La oración permanente de Adelina se constituye, de esta forma, en una práctica de sí que busca lograr un estado de suficiencia "espiritual" en el momento de "desencarnar" como desenlace posible de la enfermedad, e ir al encuentro de la divinidad. Estas prácticas de sí, no obstante, llegan a detentar aquí mismo posibilidades de ejercicio que sobrepasan la normatividad valorativa de un marco institucional.

Así mismo, según lo reflejan los relatos de Adelina y los de Blanca, la interpretación del cáncer no solo está cruzada por el principio de estructura- ción, la constitución de la experiencia y la función ideológica y práctica que le otorga la religión - a la manera de Bourdieu (2006) - o el marco de la cosmovisión con su ethos respectivo y su función de ciencia práctica - a la manera de Geertz (2005)—, sino que además las dimensiones de lo espiritual y de lo corporal (al parecer operativas en algún grado en la cultura) son abordadas igualmente y de forma particular por el sistema médico — término de Foucault (1977)—. De este modo, aquí entra a jugar "el hecho de que la existencia, la conducta, el comportamiento, el cuerpo humano, se incorporaran a partir del siglo XVIII en una red de medicalización", tal como lo señala Foucault (1977, p. 4). Esto generó una conceptualización en términos médicos tanto de la subjetividad como del cuerpo y, así mismo, unas conductas médicamente formuladas destinadas a su operación. Las ideas de Adelina sobre la herencia genética de la patología, el rol medioambiental, la alimentación y la integridad celular toman algunos de sus elementos de este proceso de medicalización del cuerpo y de la vida.

Cruzados los elementos, es importante señalar que para las mujeres - a excepción de Adelina- la espiritualidad y la religiosidad coincidían perfectamente como sinónimos y se constituían como lo mismo o, como máximo, en el caso de Blanca, la religiosidad constituía la vía conducente a la espiritualidad. Sin embargo, Blanca asociaba esta última con una cercanía especial con algunos entes mítico-religiosos propios de su adscripción religiosa: con Dios, Jesús y el Espíritu Santo. Lo mismo ocurrió con Marlén y Cecilia, quienes asociaban espiritualidad con divinidad. Adelina, por su lado, hacía explícita la distinción entre espiritualidad y religiosidad y la caracterizaba en virtud de la oposición entre una práctica moral y una práctica ritual; no obstante, las directrices de tal práctica moral venían a ser - lógicamente - las propias de su comunidad moral, es decir, las de su iglesia, siendo esta el lugar de "transfiguración de los dioses en potencias éticas" (Weber citado, por Bourdieu, 2006). 
El vínculo entre el ser humano y lo sagrado vino a ser reformulado por Weber en una división que comprendía: un código de mandamientos éticos otorgados al ser humano por la divinidad, a través de escrituras sagradas, de profetas, y la relación "directa" con la divinidad por medio de un cierto misticismo y una intuición estética (Geertz, 2005). Por supuesto, el ethos normativo de una comunidad moral conduciría al establecimiento de ciertas conductas, como las señaladas por Adelina en la forma de "obras de caridad"; pero parece también que este código de mandamientos éticos vendría a asumirse de manera parcial, y que el carácter de intermediación estaría disminuido en Adelina cuando mediante una práctica de sí estableciera un vínculo especial con "lo divino", animado por la situación de enfermedad. La particularidad de tal vínculo, en el caso de Marlén, Cecilia, Blanca y Adelina, se fundó, sin embargo, en la asociación posiblemente compartida entre "lo divino" y "lo espiritual". Así, el tipo mítico-religioso de la "divinidad" vino a desempeñar un papel preponderante y central en la interpretación y vivencia de la enfermedad.

Así era como frente a la aparición de esta última, la divinidad apenas cumplía un rol de "catalizador", que ejercía un acto indirecto de permisión (o de "no permisión" pero en todo caso de evitación de un cambio en el desenlace); mientras que en la vivencia de la enfermedad se erigía como un regente fundamental de acompañamiento del proceso. Finalmente, con respecto al rumbo del cáncer, se convertía en la causa segura de una eventual "remisión”, ya fuera por medio del ejercicio "espiritual-religioso" o de otros elementos "humanos" o "terapéuticos". En consecuencia, el papel de la espiritualidad/religiosidad en mujeres con cáncer (dadas a disposiciones de este tipo) resulta fundamental en la experiencia de la enfermedad, por cuanto posibilita su asimilación y la construcción de sentido a su alrededor.

Se considera, en últimas, que los aportes de esta investigación en relación con el entendimiento de la espiritualidad y la religiosidad en el contexto de la enfermedad - y desde la perspectiva de mujeres que la viven - contribuyen a un mejor manejo de su condición en el escenario de los sistemas de salud, de atención social y en todo contexto institucional y colectivo, razón por la que este tipo de investigaciones deben seguirse desarrollando ampliamente.

\section{Referencias}

Beltrán, W. (2010). La expansión pentecostal en Colombia: una revisión del estado del arte. En El pentecostalismo en Colombia: prácticas religiosas, liderazgo y participación política (pp. 73-96). Bogotá: CES.

Bourdieu, P. (2006). Génesis y estructura del campo religioso. Relaciones. Estudios de Historia y Sociedad, XXVII(108), 29-83.

Brady, M. J., Peterman, A. H., Fitchett, G., Mo, M., \& Cella, D. (1999). A case for including spirituality in quality of life measurement in oncology. Psychooncology, 8(5), 417-428.

Breitbart, W. (2002). Spirituality and meaning in supportive care: spirituality- and meaning-centered group psychotherapy interventions in advanced cancer. Support Care Cancer, 10(4), 272-280.

Choumanova, I., Wanat, S., Barrett, R., \& Koopman, C. (2006). Religion and spirituality in coping with breast cancer: perspectives of Chilean women. Breast Journal, 12(4), 349-352.

Csordas, T. (2004). Asymptote of the ineffable: embodiment, alterity and the theory of religion. Current Antropology, 45(2), 163-185.

Fann, J., Thomas-Rich, A., Katon, W., Cowley, D., Pepping, M., McGregor, B., \& Gralow, J. (2008). Major depression after breast cancer: a review of epidemiology and treatment. General Hospital Psychiatry-Journal, 30(2), 112-126.

Foucault, M. (1977). Historia de la medicalización. Educación Médica y Salud, 11(1), 3-25. Recuperado de http://hist.library.paho.org/Spanish/ EMS/4839.pdf 
Foucault, M. (1999). La ética del cuidado de sí como práctica de la libertad. En Estética, ética y hermenéutica: obras esenciales, volumen III (pp. 393-416). Buenos Aires: Paidós.

Geertz, C. (2005). La interpretación de las culturas: la religión como sistema cultural. Barcelona: Gedisa.

Hegel, M. T., Moore, C. P., Collins, E. D., Kearing, S., Gillock, K. L., Riggs, R. L., . . . Ahles, T. A. (2006). Distress, psychiatric syndromes, and impairment of function in women with newly diagnosed breast cancer. Cancer, 107(12), 29242931.

Instituto Nacional de Cancerología \& International Agency for Research on Cancer. (2005). Incidencia estimada y mortalidad por cáncer en Colombia, 1995-1999. Bogotá: autores.

International Agency for Research on Cancer. (2008). Globocan 2008. Recuperado de http://globocan. iarc.fr/

Karasu, T. (1999). Spiritual psychotherapy. American Journal of Psychotherapy, 53(2), 143-162.

Kim, S., Son, B., Hwang, S., Han, W., Yang, J., Lee, S., \& Yun, Y. (2008). Fatigue and depression in disease-free breast cancer survivors: prevalence, correlates, and association with quality of life. Journal of Pain and Symptom Manage, 35(6), 644-655.

Kissane, D., Bloch, S., Miach, P., Smith, G., Seddon, A., \& Keks, N. (1997). Cognitive-existential group therapy for patients with primary breast cancer--techniques and themes. Psychoonco$\log y, 6(1), 25-33$.

Mehnert, A. \& Koch, U. (2008). Psychological comorbidity and health-related quality of life and its association with awareness, utilization, and need for psychosocial support in a cancer register-based sample of long-term breast cancer survivors. Journal of Psychosomatic Research, 64(4), 383-391.

Morales, M., Robles, R., Jiménez, M., \& Morales, J. (2007). Las mujeres mexicanas con cáncer de mama presentan una alta prevalencia de depresión y ansiedad. Salud Pública de México, 49(4), 247-248.

Mytko, J. J., \& Knight, S. J. (1999). Body, mind and spirit: towards the integration of religiosity and spirituality in cancer quality of life research. Psychooncology, 8(5), 439-450.

Nairn, R. \& Merluzzi, T. (2003). The role of religious coping in adjustment to cancer. Psychooncology, 12(5), 428-441.

Piñeros, M., Ferlay, J., \& Murillo, R. (2006). Cancer incidence estimates at the national and district levels in Colombia. Salud Pública de México, 48(6), 455-465.

Puchalski, C., \& Romer, A. (2000). Taking a spiritual history allows clinicians to understand patients more fully. Journal of Palliative Medicine, 3(1), 129-137.

Rousseau, P. (2000). Spirituality and the dying patient. Journal of Clinical Oncology, 18(9), 20002002.

Swinton, J., Bain, V., Ingram, S., \& Heys, S. D. (2011). Moving inwards, moving outwards, moving upwards: the role of spirituality during the early stages of breast cancer. European Journal of Cancer Care (Engl), 20(5), 640-652.

Visser, A., Garssen, B., \& Vingerhoets, A. (2010). Spirituality and wellbeing in cancer patients: a review. Pysichooncology, 19(6), 565-572.

Zwingmann, C., Müller, C., Körber, J., \& Murken, S. (2008). Religious commitment, religious coping and anxiety: a study in German patients with breast cancer. European Journal of Cancer Care (Engl), 17(4), 361-370. 